\title{
Excess Weight Loss after Bariatric Surgery: The Influence of Catechol-0-Methyltransferase (COMT) Polymorphism (Val108/158Met), Sociodemographic Variables and Physical Activity
}

\author{
Jessica Liebaert ${ }^{1}$, Harald Rudolf Bliem¹, Christine Unterrainer ${ }^{1}$, Johann F. Kinzl ${ }^{2}$ \\ ${ }^{1}$ Department of Psychology, University of Innsbruck, Innsbruck, Austria \\ ${ }^{2}$ Department of Psychiatry, Medical University Innsbruck, Innsbruck, Austria \\ Email: ${ }^{*}$ jessica.liebaert@me.com
}

How to cite this paper: Liebaert, J., Bliem, H.R., Unterrainer, C. and Kinzl, J.F. (2020) Excess Weight Loss after Bariatric Surgery: The Influence of Catechol-O-Methyltransferase (COMT) Polymorphism (Val108/ 158Met), Sociodemographic Variables and Physical Activity. Surgical Science, 11, 194207. https://doi.org/10.4236/ss.2020.117022

Received: June 8, 2020

Accepted: July 19, 2020

Published: July 22, 2020

Copyright $\odot 2020$ by author(s) and Scientific Research Publishing Inc. This work is licensed under the Creative Commons Attribution International License (CC BY 4.0).

http://creativecommons.org/licenses/by/4.0/ Open Access

\begin{abstract}
Background: In the treatment of morbid obesity bariatric surgery has become the method of choice. Dopamine is the primary modulator of the brain's reward system and plays an essential role in the regulation of food intake. The role of dopamine is well documented in weight regulation and food intake in both animal models and humans. Still, the role of dopamine has not been well studied for weight loss. Catechol-O-methyltransferase (COMT) degrades catecholamines and estrogens are both known to be important risk factors for cardiovascular diseases and consequently obesity. The gene coding for COMT contains a Val108/158Met polymorphism that exerts a considerable influence on enzymatic activity. We hypothesized that this polymorphism might influence weight loss in obese patients, who previously underwent gastric banding surgery. In obesity research, it is known that women tend to lose more weight than men, and weight before surgery might also affect the outcome of weight loss efforts. Several studies have shown that physical activity (PA) plays an important role in maintaining weight as well as in both non-surgical and surgical weight loss. Therefore, we examined whether gender, age, weight before surgery and PA are good predictor variables for the outcome of surgical weight loss. Methods: One to six years after bariatric surgery 74 adults underwent a semi-structured interview. In a second step data on the post-surgical PA level were collected with the Moorehead-Ardelt Quality of Life Questionnaire. Finally, mouth swabs were used for genotyping. Results: 54 women and 20 men were enrolled between January 2004 and September 2009. Short-term EWL in the mid-activity genotype dopamine group (GA) was significantly
\end{abstract}


higher ( $\mathrm{p}=0.007)$ than was short-term EWL in the low-activity genotype dopamine group (AA) or in the high-activity genotype dopamine group (GG). However, there were no significant differences between the COMT groups with respect to long-term EWL. Long-term we determined that EWL is significantly positively influenced by PA and negatively by gender. The effect of EWL was more pronounced in female than in male subjects. Conclusion: Various individual genotypes of the COMT polymorphism (Val108/158Met) make an impact only on short-term EWL, but not on long-term EWL. However, gender and PA proved to be good surgical weight loss predictors for long-term EWL.

\section{Keywords}

COMT Polymorphism ( Val108/158Met), Bariatric Surgery, Excess Weight Loss (EWL), Physical Activity (PA)

\section{Introduction}

Obesity is becoming an issue of serious concern worldwide among individuals of all age groups. It is well known that bariatric surgery is an effective treatment for patients with severe obesity. Gastric banding, sleeve gastrectomy and gastric bypass have been used as the method of choice in practically $90 \%$ of bariatric surgeries [1]. Despite the fact that psychosocial factors influencing outcome after bariatric surgery (BS) have been extensively investigated, the impact of genetic polymorphisms has not. More than 120 candidate genes have been linked to obesity-related traits: Mutations in melanocortin receptor subtype 4 (MC4R), which plays a decisive role in the control of appetite and satiety, are associated with obesity. The peroxisome proliferator-activated receptor gamma (PPARG) is a transcription factor that regulates fatty acid storage und glucose metabolism in multiple ways as it activates lipid uptake and adipogenesis-stimulating genes. Polymorphisms of PPARG are implicated in the development of atherosclerosis, diabetes and obesity [2]. The fat mass and obesity-associated gene (FTO) on human chromosome 16, which is expressed in both fetal and adult tissues, is found above all in the hypothalamus, and figures into the control of feeding behavior and energy expenditure. Variants of this obesity risk allele lead to significantly higher rates of obesity as compared to persons without the risk allele [3]. As some authors see obesity as a kind of addiction to food, it is assumed that obesity and overeating share the same clinical and genetic parameters as other addictive disorders [4].

\subsection{Catechol-0-Methyltransferase (COMT)}

Catechol-O-methyltransferase is one of the main enzymes involved in catecholamine and estrogen degradation [5]. Taking into consideration that both catecholamines and estrogen are associated with changes in metabolism and food 
intake, it is hardly surprising that changes in this enzyme may contribute to weight gain [6]. There are significant indications for a link between obesity and gene polymorphisms of dopamine-metabolizing enzymes. "Eating for reward" might be associated with obesity to compensate for modified dopamine neurotransmission [7]. COMT eliminates toxic metabolites from the body and adjusts blood pressure via catecholamine metabolism. Changes in COMT activity, combined with genetic variants in the COMT gene exert effects on different mechanisms such as the development of obesity, personality changes and behavior disorders [8] [9] [10] [11].

The most explored polymorphism of the COMT gene, COMT Val108/158Met, is expressed by G-to-A transition on positions 158 - 108 of the COMT gene, leading to replacement of the amino acid valine ( $\mathrm{Val}$ ) with the amino acid methionine (Met) [12] [13]. This polymorphism is linked with a three- to fourfold variation in COMT enzyme activity, with the Val $(G)$ allele performing higher and the Met (A) allele lower enzymatic activity [14] [15].

The scientific relationship between the COMT Val108/158Met polymorphism and abdominal obesity, increased blood pressure and human hypertension is well-established [16] [17] [18]. However, no recent studies have shown an association between COMT genotype status and body mass index (BMI) [7]. The Val/Val (higher enzymatic activity) COMT genotype status is associated with greater overall food and "unhealthy" food desirability [7]. The COMT Val/Met genotype was observed to be protective for obesity into adulthood [7]. The COMT Met/Met genotype (lower enzymatic activity) was related to higher WHR [8].

The main goal of this study was to determine which individual differences in COMT polymorphism (Val108/158Met) might have an impact on the outcome of excess weight loss (EWL) in obese patients who underwent gastric banding surgery. Consistent with the findings of previous studies, we hypothesized that obese patients with the low-activity (Met/Met) genotype of dopamine would lose less weight than would the group with the high-activity (Val/Val) or the midactivity (Val/Met) genotype of dopamine.

\subsection{Sociodemographic Variables and PA}

In most studies men tend to have significantly lower weight loss and increased complication rates than do women after bariatric surgery [19] [20]. Furthermore, many studies have revealed that postoperative PA has a beneficial effect on weight loss and on maintaining it [21]. While only a few studies have carefully examined how PA has an impact on weight loss maintenance after surgery, studies of nonsurgical weight loss have demonstrated that regular PA is an important contributor to long-term weight maintenance [21]. While research studies of self-reported PA have systematically shown that patients are more active following surgery [22], it is unsure whether these increases are at least to some extent due to misperception or a reporting bias, (i.e., knowing that they should be more active, patients may feel more pressure to over-report their PA follow- 
ing surgery). Therefore, in a second focus of this study we also expected to find a difference between women and men with respect to weight reduction and we assumed that PA would show a positive influence in the process of weight reduction. Furthermore, we analyzed whether age and weight before surgery have an effect on the outcome of EWL.

\section{Patients, Materials and Methods}

In this retrospective study we examined the success rate of weight loss among severely obese patients treated with bariatric surgery at Innsbruck Medical University Hospital between 2004 and 2009. Data collection occurred in 2010. We also investigated to what extent EWL in morbidly obese patients is influenced by the different genotypes of the COMT polymorphism (Val108/158-Met), which could be a good prediction for long-term effects of weight loss. Also examined was whether other variables such as PA, gender, age, and weight before surgery have an impact on the outcome of EWL.

\subsection{Study Design and Ethics}

This was a retrospective, clinical and single-center study to evaluate the genetic and physiological state of patients who had previously undergone bariatric surgery at Innsbruck Medical University Hospital. Our study was approved by the Ethics Committee of the Medical University of Innsbruck. Patients gave written informed consent prior to participating in the study. Patient selection for bariatric surgery was performed and prior to study inclusion.

\subsection{Patients}

Adult female and male patients were diagnosed as morbidly obese by a surgeon and a psychiatrist prior to surgery. Criteria for bariatric surgery were determined, according to the guidelines of the German Obesity Society [23]. This covers the following aspects: Patients with a BMI $>40 \mathrm{~kg} / \mathrm{m}^{2}$ without side effects and contra-indications after having exhausted all possible options of conservative therapy. Patients with a BMI $>35 \mathrm{~kg} / \mathrm{m}^{2}$ with one or several obesity related implications such as type 2 diabetes mellitus, arterial hypertonia or koronare heartillness. We recruited the patients during their regular follow-up visits one to six years after their first surgery (gastric banding) at the outpatient clinic of the Department of Surgery between January 2004 and September 2009. At the beginning of this study, 113 patients were enrolled. Exclusion criteria were severe complications such as post-operative sepsis, small bowel perforation, port revision, band slippage, band obstruction, band erosion or other complications that required surgical re-intervention. Mild postoperative complications such as vomiting, abdominal pain, postoperative reflux or need for port filling did not constitute a reason for exclusion. Outcome of bariatric surgery was indicated by excess weight loss (EWL \%) and BMI reduction. Furthermore, patients had to be German-speaking and age 18 years or older. 10 pa- 
tients refused to participate in the study. 10 other patients had a gastric bypass surgery. The Moorehead-Ardelt Quality of Life Questionnaire has not been filled out completely, by 10 patients. 1 patient did not reply at all to the questionnaire. A repeat surgery was required for 5 patients. 3 patients weren't no longer available by phone or mail. Ultimately, after exclusion a total of 74 adults (54 women and 20 men) were included in this study. In order to examine EWL over the short and the long term we had to form two different categories (after bariatric surgery): those who underwent gastric banding surgery between 2008 and 2010 (at least six months and up to two years from surgery) and those between 2004 and 2007.

\subsection{Assessments}

At the follow-up visit, a nurse from the surgical department measured the height and weight of the patients and calculated their BMI. A PhD student in psychology conducted a semi-structured interview for the assessment of age, education, weight before surgery, current weight, desired weight, weight loss one year after surgery (kg, BMI), total weight loss after surgery (kg, BMI). Patients' postsurgical PA level was investigated with the Moorehead-Ardelt Quality of Life Questionnaire [24]. In addition, a mouth swab was collected by a student in order to genotype all patients for the COMT Val158 polymorphism. Genotyping was performed by the Department of Legal Medicine in accordance with the PCR protocol described in detail elsewhere.

\subsection{Excess Weight Loss: EWL (\%)}

The BMI is the best yardstick we have and it has proven to be the best way to classify patients. Nevertheless, it should be considered a rough guide because it may relate to the same degree of fat in different individuals. Therefore, EWL \% is a measuring instrument that allows patients and researchers to compare the results of various fat reduction therapies. It has become a common benchmark for surgical procedures and medical devices. The calculation is (weight loss (weight before surgery - actual weight))/(excess weight (before surgery) $) \times 100$. "Excess weight" means the difference between actual weight and the "ideal weight", calculated with the patient's height and the weight that they would need to achieve a BMI of 25. In the international literature the EWL (\%) has become a reference value and an outcome appraisal for therapy [25].

\subsection{Genotype}

The COMT polymorphism ( Val108/158Met) is known to be subdivided into three phenotypes, namely low Met-Met ((AA) this leads to a decreased reduction), intermediate mid Val-Met ((GA) this leads to normal reduction) and high Val-Val ((GG) this leads to an increased reduction in dopamine enzyme activity).

\subsection{Description of Used Questionnaires}

Data on PA were collected with an adapted version of the Moorehead-Ardelt 
Quality of Life Questionnaire (M-A QoLQ II): Self-esteem and activity levels, which is a disease-specific instrument for measuring postoperative outcomes of self-perceived quality of life (QoL). It involves six questions and measures six major domains: self-esteem (Usually I feel... very badly about myself or very good about myself), physical activity (I enjoy physical activities... not at all or very much), social relationships (I have satisfactory social contacts... none or very much), satisfaction with job (I am able to work... not at all or very much), sexual activity (the pleasure I get out of sex is... not at all or very much) and eating attitude (the way I approach food is... I live to eat or I eat to live). The question of postoperative quality of life is analyzed multi-plane. Scoring key is a 10-point Likert scale [24]. In this study, only data on postsurgical PA level were obtained with the Moorehead-Ardelt Quality of Life Questionnaire.

\subsection{Statistical Analysis}

In order to examine to what degree the surgical weight loss (EWL \%) of morbidly obese patients is influenced by the COMT polymorphism ( Val158Met) three different genotype groups were statistically built, namely with low, mid and high dopamine enzyme activity levels. Furthermore, data were divided into two groups. One group was treated with bariatric surgery between 2008 and 2009, the second group between 2004 and 2007. This division into those two segments was made in order to examine the short- and long-term effects. Data were analyzed descriptively and presented either with mean and standard deviation. One-way analyses of variance were conducted to show the main effect of the COMT groups on EWL. Post-hoc tests (Scheffé) were used for pairwise group comparisons. Multiple regression analyses were conducted to predict mean EWL (\%) with the predictors age, gender, weight before surgery and physical activity. All statistical analyses were performed with SPSS 24 (IBM, Armonk, New York, USA). The two-sided significance level was defined as $\alpha=0.05$.

\section{Results}

A total of 54 women and 20 men were enrolled between January and November 2010. All 74 patients underwent gastric banding surgery and completed the entire study protocol. Average age was 43.1 (13) years, education 9.4 (2) years and weight at patient assessment was 94.7 (19) corresponding to a BMI of 33.3 (5.2). Outcome data demonstrated an EWL of 41 (22.1) and a reduction in mean BMI of 8.8 (5.2). Between 2008 and 201021 women (77.8\%) and six men (22.2\%) underwent gastric banding surgery.

Average age was 38 (11.1) years, education 9.4 (2) years and current weight at patient assessment 97.8 (19), corresponding to a BMI of 35 (5). Outcome data demonstrated an EWL of 37 (19).

Between 2004 and 200733 women (70.2\%) and 14 (29.8\%) men underwent gastric banding surgery.

Average age was 46 (13) years, education 9.4 (2.1) years and weight at patient 
assessment 93 (18.4), corresponding to a BMI of 33 (6). Outcome data demonstrated an EWL of 43.4 (24).

Patients underwent laparoscopic gastric banding using a Swedish Adjustable Gastric Band (SAGB, Ethicon Endo-Surgery), which is a type of weight loss surgery that involves creating a small pouch from the stomach and connecting the newly created pouch precisely to the small intestine. Prior to surgery the patient sample showed the following co-morbidities: diabetes mellitus type II (45.3\%), obstructive sleep apnea (65.2\%), hypertension (18.8\%) and gastroesophageal reflux (17.2\%).

Differences in genotype: Genotype distribution is presented: the allele frequencies for the COMT Val108/158-Met polymorphism were AA (low-activity dopamine genotype group): 28 (37.8\%), GA (mid-activity genotype dopamine group): 30 (40.5\%) and GG (high-activity genotype dopamine group): 28 (37.8\%).

\subsection{Descriptive Statistics}

The descriptive statistics (Table 1) show that the means of short-term EWL in the COMT polymorphism groups differ much more than do the means of longterm EWL.

\subsection{One-Way ANOVA}

The conducted one-way analyses of variance (Table 2) revealed a strong significant main effect of the COMT groups on short-term EWL $\left(\eta^{2}=0.34, p=0.007\right)$, but not on long-term EWL $\left(\eta^{2}=0.01, p=0.890\right)$.

Table 2 illustrates the mean values of short- and long-term EWL for each COMT group as well as the results of the post-hoc-tests (Scheffé).

In keeping with our main hypothesis that obese patients with low-activity (AA) genotype of dopamine lose less weight than do the groups with high-(GG) and mid-activity (GA) genotype of dopamine, short-term EWL of the GA Group $(M=47.86)$ was significantly higher than was short-term EWL of the AA Group $(M=28.62)$ or the GG Group $(M=23.62)$. Therefore, the primary hypothesis was proven, short term. However, there were no significant differences between the COMT groups with respect to long-term EWL.

Table 1. Mean and standard deviation of EWL in the different groups of COMT polymorphism (Val158-Met).

\begin{tabular}{cccccccc}
\hline $\begin{array}{c}\text { Time after } \\
\text { Surgery }\end{array}$ & $\begin{array}{c}\text { Different } \\
\text { Genotype } \\
\text { COMT }\end{array}$ & $\mathrm{N}$ & $\begin{array}{c}\text { EWL } \\
\text { Mean (SD) }\end{array}$ & $\begin{array}{c}\text { Time after } \\
\text { Surgery }\end{array}$ & $\begin{array}{c}\text { Different } \\
\text { Genotype } \\
\text { COMT }\end{array}$ & $\mathrm{N}$ & $\begin{array}{c}\text { EWL } \\
\text { Mean (SD) }\end{array}$ \\
\hline $\begin{array}{c}2008-2010 \\
\text { (short-term) }\end{array}$ & AA & 8 & $28.62(15.27)$ & $\begin{array}{c}2004-2007 \\
\text { (long-term) }\end{array}$ & AA & 20 & $45.35(29.43)$ \\
& GG & 6 & $23.62(12.72)$ & & GA & 17 & $41.78(16.09)$ \\
& TOTAL & 27 & $36.77(18.90)$ & & TOTAL & 47 & $43.39(23.55)$ \\
\hline
\end{tabular}

Note: $N=74$; AA: low reduction in dopamine; GA: normal reduction in dopamine; GG: high reduction in dopamine; ${ }^{*} \mathrm{p}<0.05^{* *} \mathrm{p}<0.01{ }^{* * *} \mathrm{p}<0.001$. 
Table 2. One-way analysis of variance (COMT) for EWL excess weight loss \%.

\begin{tabular}{cccc}
\hline COMT group & $\begin{array}{c}\text { Short-term EWL } \\
\text { Time after surgery } \\
(2008-2010)\end{array}$ & COMT group & $\begin{array}{c}\text { Long-term EWL } \\
\text { Time after surgery } \\
(2004-2007)\end{array}$ \\
\hline AA, Group I $(n=8)$ & $28.62^{\mathrm{II}}$ & AA Group I $(n=20)$ & 45.35 \\
GA, Group II $(n=13)$ & $47.86^{\mathrm{IIII}}$ & GA Group II $(n=17)$ & 41.78 \\
GG, Group III $(n=6)$ & $23.62^{\mathrm{II}}$ & GG Group III $(n=10)$ & 42.21 \\
Sum of squares & 3169.30 & Sum of squares & 134.96 \\
$d f$ & 2 & $d f$ & 2 \\
Mean squares & 1584.65 & Mean squares & 67.48 \\
$F$ & 6.21 & $F$ & 0.12 \\
$p$ & 0.007 & $p$ & 0.890 \\
$\eta^{2}$ & 0.34 & $\eta^{2}$ & 0.01 \\
\hline
\end{tabular}

Notes: $\eta^{2}=$ partial eta square; I, II, III: significant differences (Scheffé post-hoc test) to each of the named groups.

Table 3. Influence of gender, age, weight before surgery and PA on the outcome of EWL \% (dependent variable).

\begin{tabular}{ccccccc}
\hline $\begin{array}{c}\text { Time after } \\
\text { Surgery }\end{array}$ & & B & SE & Beta & T & P \\
\hline \multirow{2}{*}{$2008-2010$} & Gender & -6.098 & 10.480 & -0.137 & -0.582 & 0.567 \\
Short-term EWL & Age & -0.453 & 0.304 & -0.267 & -1.493 & 0.150 \\
& Weight before surgery & 0.330 & 0.218 & 0.345 & 1.515 & 0.144 \\
& PA & 29.311 & 18.760 & 0.310 & 1.562 & 0.132 \\
$2004-2007$ & Gender & -16.328 & 7.084 & -0.321 & -2.305 & 0.026 \\
Long-term EWL & Age & 0.244 & 0.284 & -0.133 & 0.861 & 0.394 \\
& Weight before surgery & 0.358 & 0.223 & 0.239 & 1.600 & 0.117 \\
& PA & 61.302 & 16.495 & 0.500 & 3.716 & 0.001 \\
\hline
\end{tabular}

Note: $\mathrm{N}=74 ;{ }^{*} \mathrm{p}<0.05 ;{ }^{* *} \mathrm{p}<0.01 ;{ }^{* * *} \mathrm{p}<0.001$.

Additionally, multiple regression analyses were performed to investigate whether gender, age, weight before surgery and PA were related to short-term and long-term EWL. Table 3 shows that neither sociodemographic data nor PA predict short-term EWL. However, long-term EWL was significantly related to PA (positively) and gender (negatively). Women showed higher EWL than did men.

\section{Discussion}

In this retrospective clinical study we researched the impact of genetic variations in the dopamine transporter gene as well as of PA and sociodemographic data such as gender, age, weight before surgery, on the short- and long-term outcome of EWL in morbidly obese patients following bariatric surgery. 


\subsection{Catechol-0-Methyltransferase (COMT)}

In keeping with our main hypothesis short-term EWL in the GA (Val/Met) Group ( $M=47.86)$ was significantly higher than was short-term EWL in the AA (Val/ Val) Group $(M=28.62)$ or the GG (Met/Met) Group $(M=23.62)$. We were able to partly confirm our hypothesis (short-term), but significant differences between dopamine genotype groups could not be proven long-term. These findings were consistent with those of other scientific studies. The Met allele is known to be less active than the Val allele also with respect to estrogen degradation [5]. In another scientific paper an association between COMT Val158-Met polymorphism and waist-to-hip ratio (WHR) was established in middle-aged Swedish men [17]. The Met allele was related to higher WHR in a dose-dependent manner. This current scientific knowledge including the fact that there was a significant correlation between the studied polymorphism and WHR, but not BMI, supports the view that weight and fat distribution are modulated by different genes [7] [17] [18]. Unfortunately, we had no access to patients' WHR in our study, thus making a comparison of results difficult. A previous other study published by Hong and coworkers observed in 350 women a relation between the COMT Val158Met polymorphism and BMI in the premenopausal group; in fact, this association was weaker than that noticed for WHR and BMI and disappeared after adjustment for levels of growth hormone and insulin-like growth factor 1 [26]. In fact, WHR is one of a number of risk factors for breast cancer. A different study by Happonen et al. observed no impact of the COMT Val158-Met polymorphism and WHR [27]. Also, the Met allele of the COMT Val158-Met polymorphism was found to be an independent risk factor for cardiovascular disorders (associated with higher blood pressure) in men [17]. Two recent Finnish studies with large sample size show that Met carriers increase the risk for acute coronary events [27] [28].

\subsection{Sociodemographic Data and PA}

We observed that neither sociodemographic data nor PA were predictors for EWL over the short term. Over the long term, we found that EWL is significantly negatively influenced by gender and positively by PA. Women showed a higher EWL than did men. This result is consistent with the finding made in most recent studies, namely that men tend to have significantly less weight loss and increased complication rates. Moreover, they also have higher satisfaction and psychological well-being scores than do females [18]. It should also be noted that the majority of the participants in clinical studies and weight-loss programs are women. Almost $80 \%$ of all bariatric surgery patients are female [19]. Likewise, our study involved more women than men. Women are more insulin-sensitive and have more subcutaneous fat, while men have more visceral and liver fat and higher cardiovascular risk. Women more often present the metabolically healthy obese phenotype than do men [19]. The reason we chose to examine the role of 
PA in weight loss in this study is because PA enhances metabolic rate via its effect on building or maintaining lean body tissue and stimulating individual basal metabolic rate following PA. Furthermore, we would like to point out that regular PA also alters fat distribution by selectively mobilizing central fat, improving the body's ability to burn fat, and decreasing fat cell size. Consequently, PA is an important cooperator for weight management. Whereas the main goal of BS is to decrease initial body mass, especially body fat, it also leads to lean body mass (e.g., muscle, bone density), in particular during the period of quick weight loss shortly after BS [29]. Many studies demonstrate change in PA pre- to one year post-surgery. For example, Bond et al. (2009) [30] showed that 68 (34\%) of 199 patients went from an inactive to an active and nine (5\%) from an active to an inactive lifestyle, while most were either active $(n=83 ; 42 \%)$ or inactive $(n=39$; $20 \%$ ) at both measuring points. Together, these clinical trials suggest that not only will a few patients fail to improve their PA, but some of them will become less active one year after bariatric surgery [29]. We found that the more physical activity (self-reported PA) patients undertake, the better their weight loss will be within three to six years from bariatric surgery. Our study shows that a more active lifestyle has an influence on long-term outcome of weight loss, but that from a short-time perspective patients seem to have no weight loss benefits. Similar results were found in a study of 108 adolescents with severe obesity, namely from baseline to three years after metabolic bariatric surgery (MBS). Increased activity in adolescents was related to improvements in cardiovascular diseaserelated lipid measures and weight loss after MBS [31]. A recent study analyzed PA and sitting time in 398 BS patients one to 16 years post-surgery and assessed their associations with patient, surgery and weight-loss characteristics. Patients completed a telephone questionnaire. The results showed that over the long-term following surgery there was low PA and high sedentariness among bariatric patients. There was a need to promote active lifestyle in this patient population in order to maintain weight management over the long-term following surgery [32]. Our results support this need. Consequently, we recommend close cooperation between surgeons, psychiatrists, psychologists and nutritionists in order to provide the best possible post-operative care following bariatric surgery. In conclusion, our results reveal that in the short term the carriers of the combined AG genotypes achieved significantly better weight loss than did the carriers of the combined AA or GG genotypes although this effect was not able to be confirmed over the long term. Future studies exploring the outcome of bariatric surgery should consider the genetic influence of the COMT Val158-Met polymorphism as well as the need for gender and post-operative physical activity. Further understanding of this relationship is needed for the development of post-surgical weight loss guidelines and interventions. Furthermore, it is key that the bariatric team explain that PA, along with surgery, is a tool for promoting weight loss and improved health as well as for preventing weight regain. An appropriate PA program should be formulated that suits patients' needs and lifestyle. 


\section{Limitations}

This study has several limitations, including the fact that its design was retrospective and that it was conducted in a single center. This clinical study had no obese healthy control group for the purpose of comparison. The reliability of data shown may be affected by the small sample size. Conclusions may also be limited by the small sample size although we were able to enroll enough patients to obtain significant differences. In addition, the single-center study design allowed us to diminish inter-investigator variability and differences in clinical procedures. Another limitation is the fact that data regarding waist and hip circumference were not available and therefore it was not possible to calculate metabolic syndrome with the currently used criteria. One more limitation of this study was that patients were enrolled at their regular visit at the outpatient clinic of the Department of Surgery. Thus, we cannot rule out the possibility of a selection bias imposed by the lack of data on patients with severe complications or by non-compliant patients. Additionally, we were faced with the problem that some patients refused to participate in the study. It seems that these patients had experienced little therapeutic success or a failure to lose weight. As the sample size was small for a genetic study, we present our results regarding the influence of the COMT Val 158-Met polymorphisms with caution. Nonetheless, in future such investigations should involve larger samples of post-bariatric patients.

\section{Fund}

This study was financially supported by Fonds National de la Recherche Luxembourg.

\section{Ethical Approval}

The study was conducted to the principles of the Declaration of Helsinki and was approved by the Ethics Committee of the Medical University of Innsbruck.

\section{Conflicts of Interest}

The authors declare no conflicts of interest regarding the publication of this paper.

\section{References}

[1] O’Brien, P.E. (2010) Bariatric Surgery: Mechanisms, Indications and Outcomes. Journal of Gastroenterology and Hepatology, 8, 1358-1365. https://doi.org/10.1111/j.1440-1746.2010.06391.x

[2] Razquin, C., Martin, A. and Martinez, J.A. (2001) Evidences on Three Relevant Obesogenes. Molecular Nutrition \& Food Research, 55, 136-149. https://doi.org/10.1002/mnfr.201000445

[3] Fawcett, K.A. and Barroso, I. (2010) The Genetics of Obesity: FTO Leads the Way. Trends in Genetics, 26, 266-274. https://doi.org/10.1016/j.tig.2010.02.006

[4] Kinzl, J.F. (2010) Morbid Obesity: Significance of Psychological Treatment after Ba- 
riatric Surgery. Eating and Weight Disorders, 15, e275-e280.

[5] Creveling, C.R. (2003) The Role of Catechol-O-Methyltransferase in the Inactivation of Catecholestrogen. Cellular and Molecular Neurobiology, 23, 289-291. https://doi.org/10.1023/A:1023680302975

[6] Halford, J.C.G., Cooper, G.D. and Dovey, T.M. (2004) The Pharmacology of Human Appetite Expression. Current Drug Targets, 5, 221-240. https://doi.org/10.2174/1389450043490541

[7] Wallace, D.L., Aarts, E., d'Oleire Uquillas, F., Dang, L.C., Greer, S.M., Jagust, W.J. and D'Esposito, M. (2015) Genotype Status of the Dopamine-Related Catechol- $O$ Methyltransferase (COMT) Gene Corresponds with Desirability of "Unhealthy" Foods. Appetite, 92, 74-80. https://doi.org/10.1016/j.appet.2015.05.004

[8] Nedic, G., Nikolac, M., Nenadic Sviglin, K., Muck-Seler, D., Borovecki, F. and Pivac, N. (2001) Association Study of Functional Catechol-O-Methyltransferase (COMT) $\mathrm{Val}^{108 / 158}$ Met Polymorphism and Suicide Attempts in Patients with Alcohol Dependence. International Journal of Neuropsychopharmacology, 14, 377-388. https://doi.org/10.1017/S1461145710001057

[9] Nedic, G., Borovecki, F., Klepac, N., Mubrin, Z., Hajnsek, S., Nikolac, M., MuckSeler, D. and Pivac, N. (2011) Association Study of a Functional Catechol-O-Methyltransferase Polymorphism and Cognitive Function in Patients with Dementia. Collegium Antropologicum, 35, 79-84.

[10] Malhotra, A.K., Lencz, T., Correll, C.U. and Kane, J.M. (2007) Genomics and the Future of Pharmacotherapy in Psychiatry. International Review of Psychiatry, 19, 523-530. https://doi.org/10.1080/09540260701563460

[11] Nedic Erjavec, G., Nenadic Sviglin, K., Nikolac Perkovic, M., Muck-Seler, D., Jovanovic, T. and Pivac, N. (2014) Association of Gene Polymorphisms Encoding Dopaminergic System Components and Platelet MAO-B Activity with Alcohol Dependence and Alcohol Dependence-Related Phenotypes. Progress in Neuro-Psychopharmacology and Biological Psychiatry, 54, 321-327. https://doi.org/10.1016/j.pnpbp.2014.07.002

[12] Lachman, H.M., Papolos, D.F., Saito, T., Yu, Y.M., Szumlanski, C.L. and Weinshilboum, R.M. (1996) Human Catechol-O-Methyltransferase Pharmacogenetics: Description of a Functional Polymorphism and Its Potential Application to Neuropsychiatric Disorders. Pharmacogenetics, 6, 243-250. https://doi.org/10.1097/00008571-199606000-00007

[13] Lotta, T., Vidgren, J., Tilgmann, C., Ulmanen, I., Melen, K., Julkunen, I. and Taskinen, J. (1995) Kinetics of Human Soluble and Membrane-Bound Catechol-O-Methyltransferase: A Revised Mechanism and Description of the Thermolabile Variant of the Enzyme. Biochemistry, 34, 4202-4210. https://doi.org/10.1021/bi00013a008

[14] Chen, J., Lipska, B.K., Halim, N., Ma, Q.D., Matsumoto, M., Melhem, S., et al. (2004) Functional Analysis of Genetic Variation in Catechol-O-Methyltransferase (COMT): Effects on mRNA, Protein, and Enzyme Activity in Postmortem Humain Brain. The American Journal of Human Genetics, 75, 807-821. https://doi.org/10.1086/425589

[15] González-Castro, T.B., Hernández-Díaz, Juráez-Rojop, I.E., Lòpez-Narváez, M.L., Tovilla-Zárate, C.A. and Fresan, A. (2016) The Role of Catechol-O-Methyltransferase (COMT) Val158Met Genetic Polymorphism in Schizophrenia: A Systematic Review and Updated Meta-Analysis on 32,816 Subjects. NeuroMolecular Medicine, 18, 216 231. https://doi.org/10.1007/s12017-016-8392-Z

[16] Avsar, O., Kuskucu, A., Sancak, S. and Genc, E. (2017) Are Dopaminergic Geno- 
types Risk Factors for Eating Behavior and Obesity in Adults? Neuroscience Letters, 654, 28-32. https://doi.org/10.1016/j.neulet.2017.06.023

[17] Annerbrink, K., Westberg, L., Nilsson, S., Rosmond, R., Holm, G. and Eriksson, E. (2008) Catechol-O-Methyltransferase val158-Met Polymorphism Is Associated with Abdominal Obesity and Blood Pressure in Men. Metabolism-Clinical and Experimental, 57, 708-711. https://doi.org/10.1016/j.metabol.2008.01.012

[18] Kamide, K., Kokubo, Y., Yang, J., Matayoshi, T., Inamoto, N., Takiuchi, S., et al. (2007) Association of Genetic Polymorphisms of ACADSB and COMT with Human Hypertension. Journal of Hypertension, 25, 103-110. https://doi.org/10.1097/HJH.0b013e3280103a40

[19] Kochkodan, J., Telem, D.A. and Ghaferi, A.A. (2018) Physiologic and Psychological Gender Differences in Bariatric Surgery. Surgical Endoscopy, 32, 1382-1388. https://doi.org/10.1007/s00464-017-5819-Z

[20] Kautzky-Willer, A. (2016) Brennpunkt Adipositas: What Gender Has to Do with...! Wiener Medizinische Wochenschrift, 166, 75-78. https://doi.org/10.1007/s10354-016-0440-7

[21] Rippe, J.M. and Hess, S. (1998) The Role of Physical Activity in the Prevention and Management of Obesity. Journal of the Academy of Nutrition and Dietetics, 98, S31-S38. https://doi.org/10.1016/S0002-8223(98)00708-1

[22] Jacobi, D., Ciangura, C., Couet, C. and Oppert, J.M. (2011) Physical Activity and Weight Loss Following Bariatric Surgery. Obesity Reviews, 12, 366-377.

https://doi.org/10.1111/j.1467-789X.2010.00731.x

[23] Dag, D.D. (2014) Interdisziplinäre Leitlinie der Qualität S3 zur "Prävention und Therapie der Adipositas". Deutsche Adipositas Gesellschaft, Deutsche Gesellschaft für Ernährung, Deutsche Gesellschaft für Ernährungsmedizin, Version 2.0.

[24] Moorehead, M.K., Ardelt-Gattinger, E., Lechner, H., et al. (2003) The Validation of the Moorehead-Ardelt Quality of Life Questionnaire II. Obesity Surgery, 5, 684-692. https://doi.org/10.1381/096089203322509237

[25] Deitel, M.E. and Greenstein, R.J. (2003) Recommendations for Reporting Weight Loss. Obesity Surgery, 13, 159-160. https://doi.org/10.1381/096089203764467117

[26] Hong, C.C., Thompson, H.J., Jiang, C., Hammond, G.L., Trichler, D., Yaffe, M., et al. (2003) Val ${ }^{158}$ Met Polymorphism in Catechol-O-Methyltransferase Gene Associated with Risk Factors for Breast Cancer. Cancer Epidemiology, Biomarkers \& Prevention, 12, 838-847.

[27] Happonen, P., Voutilainen, S., Tuomainen, T.P. and Salonen, J.T. (2006) Catechol$O$-Methyltransferase Gene Polymorphism Modifies the Effect of Coffee Intake on Incidence of Acute Coronary Events. PLOS ONE, 1, e117.

https://doi.org/10.1371/journal.pone.0000117

[28] Voutilainen, S., Tuomainen, T.P., Korhonen, M., Mursu, J., Virtanen, J.K., Happonen, P., et al. (2007) Functional COMT Val ${ }^{158}$ Met Polymorphism, Risk of Acute Coronary Events and Serum Homocysteine: The Kuopio Ischaemic Heart Disease Risk Factor Study. PLOS ONE, 2, e181. https://doi.org/10.1371/journal.pone.0000181

[29] Metcalf, B., Rabkin, R.A., Rabkin, J.M., Metcalf, L. and Lehman-Becker, L.B. (2005) Weight Loss Composition: The Effects of Exercise Following Obesity Surgery as Measured by Bioelectrical Impedance Analysis. Obesity Surgery, 15, 183-186. https://doi.org/10.1381/0960892053268381

[30] Bond, D.S., Phelan, S., Leahey, T.M., Hill, J.O. and Wing, R.R. (2009) Weight-Loss Maintenance in Successful Weight Losers: Surgical vs Non-Surgical Methods. Inter- 
national Journal of Obesity, 33, 173-180. https://doi.org/10.1038/ijo.2008.256

[31] Price, P.H., Kaizer, A.M., Daniels, S.R., Jenkins, T.M., Inge, T.H. and Eckel, R.H. (2019) Physical Activity Improves Lipid and Weight-Loss Outcomes After Metabolic Bariatric Surgery in Adolescents with Severe Obesity. Obesity, 27, 989-996. https://doi.org/10.1002/oby.22475

[32] Herman, K.M., Carver, T.E., Christou, N.V. and Andersen, R.E. (2014) Physical Activity and Sitting Time in Bariatric Surgery Patients 1 - 16 Years Post-Surgery. Clinical Obesity, 4, 267-276. https://doi.org/10.1111/cob.12069 